This is the post print version of the article, which has been published in Journal of affective disorders. 2018, 236, 252-258. http://dx.doi.org/10.1016/j.jad.2018.04.084 .

\title{
Temperament clusters associate with anxiety disorder comorbidity in depression
}

Vesa Paavonen ${ }^{\mathrm{a} 1}$, Kaisa Luoto ${ }^{\mathrm{a}}$, Antero Lassila $^{\mathrm{c}}$, Esa Leinonen $^{\mathrm{a}, \mathrm{b}}$, Olli Kampman ${ }^{\mathrm{a}, \mathrm{c}}$

a University of Tampere, Faculty of Medicine and Life Sciences, FI-33014 Tampere, Finland

b Tampere University Hospital, Department of Psychiatry, FI-33014 Tampere, Finland

c Seinäjoki Hospital District, Department of Psychiatry, Huhtalantie 53, 60220 Seinäjoki, Finland 


\section{Abstract}

Background: Individual temperament is associated with psychiatric morbidity and could explain differences in psychiatric comorbidities. We investigated the association of temperament profile clusters with anxiety disorder comorbidity in patients with depression.

Methods: We assessed the temperament of 204 specialized care-treated depressed patients with the Temperament and Character Inventory (TCl-R) and their diagnoses with the Mini International Neuropsychiatric Interview. Two-step cluster analysis was used for defining patients' temperament profiles and logistic regression analysis was used for predicting different anxiety disorders for various temperament profiles.

Results: Four temperament clusters were found: 1) Novelty seekers with highest Novelty Seeking scores $(n=56), 2)$ Persistent with highest Persistence scores $(n=36), 3)$ Reserved with lowest Novelty Seeking scores ( $n=66)$ and 4) Wearied with highest Harm avoidance, lowest Reward Dependence and lowest Persistence scores $(n=58)$. After adjusting for clinical variables, panic disorder and/or agoraphobia were predicted by Novelty seekers' temperament profile with odds ratio $[\mathrm{OR}]=3.5(95 \%$ confidence interval $[\mathrm{Cl}]=1.8-6.9$, $p<0.001)$, social anxiety disorder was predicted by Wearied temperament profile with $\mathrm{OR}=3.4(95 \% \mathrm{Cl}=1.6-$ 7.5, $p=0.002$ ), and generalized anxiety disorder was predicted by Reserved temperament profile with $\mathrm{OR}=2.6(95 \% \mathrm{Cl}=1.2-5.3, p=0.01)$.

Limitations: The patients' temperament profiles were assessed while displaying depressive symptoms, which may have affected results.

Conclusions: Temperament clusters with unique dimensional profiles were specifically associated with different anxiety disorders in this study. These results suggest that TCI-R could offer a valuable dimensional method for predicting the risk of anxiety disorders in diverse depressed patients.

Keywords: depression; generalized anxiety disorder; social anxiety disorder; panic disorder; temperament; cluster analysis 


\section{Introduction ${ }^{2}$}

The comorbidity between depressive and anxiety disorders is marked and co-occurrence of more than one disorder is more of a rule than an exception (Brown et al., 2001; Melartin et al., 2002). In clinical populations, comorbidity proportions of different anxiety disorders in major depressive disorder (MDD) vary from $2 \%$ to $41 \%$ and co-occurrence of at least two different anxiety disorders vary from $36 \%$ to $64 \%$ between different disorders (Brown et al., 2001). Comorbid anxiety disorders can lead to increased treatment resistance and chronic depression (Burcusa and lacono, 2007; Holzel et al., 2011) and better comprehension of the etiology of these disorders could enable development of more efficient treatments than currently available ones. The present categorical classification system of psychiatric disorders has been criticized because of a high rate of comorbidity of different diagnoses (Naragon-Gainey et al., 2016). Certain temperamental traits have been suggested to be contributors of a dimensional diagnostic model because they could account for shared symptomatology in both depression and anxiety disorders (Barlow and Kennedy, 2016; Craske and Waters, 2005).

Whereas human temperament is generally associated with individual life choices and behavior (Al-Halabi et al., 2010; Bereczkei and Czibor, 2014; Campbell et al., 2013; El Sheikh et al., 2014; Otani et al., 2008), skewed temperament profile is also associated with psychiatric disorders (Miettunen and Raevuori, 2012). The most important model developed from the clinical point of view and in part based on biological studies for characterizing human temperament is the psychobiological model of temperament and character (Cloninger, 1986; Cloninger et al., 1993). According to this model, human temperament is largely heritable and is a relatively stable part of personality. Human temperament is considered to present a precognitive bias reflected as individual tendency to 1) avoid possible adverse or threatening situations (Harm Avoidance, HA), 2) participate in events and activities for the sake of novelty and thrill (Novelty Seeking,

\footnotetext{
${ }^{2}$ Abbreviations: $\mathrm{MDD}=$ major depressive disorder, $\mathrm{TCl}-\mathrm{R}=$ Temperament and Character Inventory, NS = Novelty Seeking, $\mathrm{HA}=$ Harm Avoidance, $\mathrm{RD}=$ Reward Dependence, $\mathrm{P}=$ Persistence, $\mathrm{PDA}=$ panic disorder or/and agoraphobia, $\mathrm{SAD}=$ social anxiety disorder, $\mathrm{GAD}$ = generalized anxiety disorder, MADRS = Montgomery-Åsberg Depression Rating Scale, AUDIT = Alcohol Use Disorder Identification Test, MINI = The Mini International Neuropsychiatric Interview
} 
NS), 3) seek social approval of others (Reward Dependence, RD), and 4) be persistent in ones efforts, even in the absence of imminent rewards (Persistence, P) (Cloninger et al., 1993). Although the associations between temperament and affective disorders are most likely complex (Klein et al., 2011), certain temperament traits could predispose to psychopathology via disadvantageous cognitions (e.g., rumination) (Mezulis et al., 2011) and behaviors (e.g., avoidant behavior or excessive reassurance seeking) (Cheavens and Heiy, 2011; Kanter et al., 2008; Weinstock and Whisman, 2007).

The temperament trait 'high $\mathrm{HA}^{\prime}$ is associated with MDD and all anxiety disorders. HA and depression severity are positively correlated and social phobia or social anxiety disorder (SAD) appear to be associated with higher levels of HA than other anxiety disorders (Kampman and Poutanen, 2011; Kampman et al., 2014; Miettunen and Raevuori, 2012). Indeed, the trait 'high HA' could be an important common etiological factor for depression and anxiety, and according to Cloninger's original theory, it responds to "obsessional" information-processing type associated with "cognitive anxiety" (Cloninger, 1986). Although Cloninger also postulated that high Novelty Seeking would respond to "histrionic" information-processing type associated with "somatic anxiety", (Cloninger, 1986) studies have not systematically supported associations between this trait and different anxiety disorders. Instead, high NS is associated with substance use disorders (SUDs) and dual diagnosis (Fernandez-Mondragon and Adan, 2015; Howard et al., 1997; Sher et al., 2000), whereas intermediate and low levels of NS have been found in panic disorder and in SAD, respectively (Kampman et al., 2014; Miettunen and Raevuori, 2012). The trait 'high RD' could be associated with panic disorder, but the results are incongruent, with different directions observed for the association between genders (low RD for women and high RD for men) (Kampman et al., 2014; Starcevic et al., 1996). In patients with social anxiety disorder, results regarding RD have also been mixed and are on average at an intermediate level according to one meta-analysis (Kampman et al., 2014). For P patients with anxiety disorders, intermediate scores have generally been observed, while low scores have shown to be associated with social phobia and alcohol dependence among patients without anxiety disorders (Kampman et al., 2014; Miettunen and Raevuori, 2012; Rae et al., 2002). GAD has been associated with high HA and impulsive symptoms in patients with GAD could be associated with high NS and low RD (Kampman et al., 2014; Piero, 2010). 
Although meta-analytic data exist on the association between separate temperament dimensions and different anxiety disorders, no studies have investigated the impact of temperament clusters on occurrence of anxiety disorders. Individual profile data on all temperament dimensions have been suggested to possibly offer more comprehensive information of the association between temperament and different anxiety disorders (Miettunen and Raevuori, 2012). In this study, we aim to explore if temperament profiles determined with cluster analysis are associated with the likelihood of anxiety disorders (panic disorder and/or agoraphobia [PDA], social anxiety disorder and generalized anxiety disorder) in a sample of depressed patients.

\section{Methods}

\subsection{Participants}

In brief, the study group comprised 242 patients who were referred to psychiatric services because of depressive symptoms, anxiety, self-destructiveness, insomnia or substance-related problems. Patients scoring a minimum of 17 points on the Beck Depression Inventory (BDI, version 1A, (Beck et al., 1996) and thus reflecting at least a moderate level of depressive symptoms, were included in the study. Patients with organic brain disease or psychotic (ICD-10 F2*) disorder were excluded from the study. A description of the study protocol and the screened patient sample has been presented elsewhere (see ClinicalTrials.gov Identifier NCT02520271, Ostrobothnia Depression Study [ODS], 2016 and (Paavonen et al., 2016)). Up to 38 (15.7\%) patients dropped out from the study after enrollment, and 204 patients (out of which 120 (59\%) were women) were included in the final analysis. Sociodemographic data, MADRS and AUDIT-C scores of the patient sample are available as supplementary electronic background material.

\subsection{Procedures}

Included patients completed the forms for the following assessments: The Temperament and Character Inventory (TCI-R) (Cloninger et al., 1993), and Alcohol Use Disorders Identification Test (AUDIT-C [questions 1-3 of AUDIT questionnaire]) (Bohn et al., 1995). Sociodemographic data were also collected. The clinical evaluation included the Montgomery-Åsberg Depression Rating Scale (MADRS) (Montgomery and Asberg, 
1979), the Mini International Neuropsychiatric Interview 5.0 (MINI) (Sheehan et al., 1998), and patients' medication was also evaluated. The antidepressant doses prescribed to each patient were converted to fluoxetine equivalents for enabling comparison of prescribed antidepressants between different patient groups, which has been described in further detail elsewhere (Paavonen et al., 2016).

According to the MINI, 181 patients (88.7\%) met the criteria for MDD, 17 (8.3\%) for dysthymia, 25 (12.3\%) for hypomanic episode (life-time), 49 (24\%) for panic disorder, 35 (17.2\%) for agoraphobia, 40 (19.6\%) for social anxiety disorder, 68 (33.3\%) for generalized anxiety disorder, 15 (7.4\%) for obsessive compulsive disorder, 15 (7.4\%) for post-traumatic stress disorder, 66 (32.4\%) for alcohol use disorder, 10 (4.9\%) for other substance use disorder and 5 (2.5\%) for bulimia. Comorbidity of disorders was marked and 150 (74\%) patients were diagnosed with at least two disorders. As was expected, co-occurrence of panic disorder and agoraphobia was marked and 69 (33.8\%) patients had either panic disorder or agoraphobia or both. An overlap was also observed for GAD with other anxiety disorders (PD, AP, OCD, SAD, PTSD) and 41 patients (20.1\%) had GAD without these comorbidities. In this study, we focused on the four most prevalent anxiety disorders in the sample: panic disorder and/or agoraphobia (PDA), SAD and GAD.

\subsection{Ethical issues}

The study was approved by the local Human Subjects Review Committee and participants gave their informed written consent. All included patients were evaluated as having the capacity to give informed consent because patients with psychotic and organic brain diseases were excluded. For further information, see ClinicalTrials.gov Identifier (NCT02520271).

\subsection{Statistical methods}

A two-step cluster analysis was used for defining patients' temperament profiles. This method allows handling of large data sets by first identifying groupings with quick cluster algorithm (pre-clustering) and it runs hierarchical cluster models in the second step. Temperament dimension scores of NS, HA, RD and P were used in the cluster model. Number of clusters were set to automatic for achieving natural clustering. 
The differences in discrete variables (age, antidepressant dose, AUDIT-C score and MADRS score) between temperament clusters were calculated with ANOVA. Differences between grouping variables (gender, the use of antipsychotics, and diagnoses) were calculated with $\chi^{2}$-statistics.

To adjust for possible confounding variables (age, gender, depression severity and alcohol use) binary logistic regression models were used for predicting the following diagnoses: 1) panic disorder and/or agoraphobia (PDA), 2) social anxiety disorder (SAD) and 3) generalized anxiety disorder (GAD). The diagnoses were predicted with the temperament cluster which had the highest prevalence of the dependent diagnosis (these prevalences are reported in Table 1.) and the models were adjusted with 1) age and gender in Model 1, 2) age, gender and MADRS scores in Model 2, and 3) age, gender, MADRS and AUDIT-C scores in Model 3. The used explanatory (binary) temperament cluster variables were: 1) Novelty seekers cluster vs. others in models predicting PDA, 2) Wearied cluster vs. others in models predicting SAD, and 3) Reserved cluster vs. others in models predicting GAD. All analyses were performed with SPSS for Mac (version 24.0, IBM Inc. Armonk, New York, USA).

\section{Results}

Based on $\mathrm{TCl}-\mathrm{R}$ temperament dimension scores, the two-step cluster analysis produced four temperament clusters with fair overall quality. After their assessment, these four temperament clusters were named with descriptive labels according to the temperament profile reflecting the combination of scores in different temperament dimensions: 1 ) Novelty seekers with highest NS, $n=56$, (NS=119.4 $\pm 9.0, H A=111.0 \pm 14.2$, $R D=103.3 \pm 13.5, P=98.0 \pm 15.5$; mean $\pm S D), 2$ ) Persistent with lowest $H A$ and highest $P, n=36$, (NS=99.6 \pm 17.1 , $H A=87.3 \pm 11.0, R D=107.9 \pm 15.6, P=125.0 \pm 12.6), 3)$ Reserved with lowest $N S, n=66$, (NS=88.1 \pm 12.3 , $H A=119.9 \pm 11.5, R D=104.5 \pm 14.6, P=105.4 \pm 15.6)$, and 4) Wearied with highest $H A$ and lowest $R D$ and $P$, $n=58,(N S=95.7 \pm 10.4, H A=129.8 \pm 14.8, R D=84.7 \pm 14.3, P=82.4 \pm 15.3)$.

[Figure 1.]

No differences were observed between the temperament clusters in gender distribution, depression severity, alcohol use or in the use of antipsychotics or prescribed antidepressant doses (Table 1). Persistent 
and Reserved patients were younger compared with Wearied patients. The Persistent cluster had the lowest occurrence of all anxiety disorders and PDA was most prevalent in Novelty seekers, SAD in Wearied and GAD in Reserved. The prevalence of different diagnoses in each of the temperament clusters of and $\chi^{2}$ and $p$ values for the differences in the occurrence of the disorders between clusters are presented in the Table 1.

\section{[Table 1.]}

The Novelty seekers cluster had higher odds (odds ratio $[O R]=3.28-3.52, p \leq 0.001$ ) for PDA compared with other patients in the three logistic regression models adjusted with 1) gender and age, 2) gender, age and depression severity, and 3) gender, age, depression severity and alcohol use. In the three models explaining $S A D$, the Wearied cluster had higher odds ( $O R=3.17-3.41, p=0.002-0.003)$ for SAD compared with other patients and in the three models explaining GAD, the Reserved cluster had higher odds (OR=2.53-2.60, $p=0.009-0.01)$ for GAD compared with the other patients. The results of these regression models are presented in Table 2.

[Table 2.]

\section{Discussion}

Our main findings were that Novelty seekers, Reserved and Wearied temperament clusters were uniquely associated with different anxiety disorders, whereas the Persistent temperament cluster was associated with significantly lower prevalence of anxiety disorders. More specifically, panic disorder and/or agoraphobia were predicted by the Novelty seekers temperament, social anxiety disorder by Wearied temperament and generalized anxiety disorder by Reserved temperament in this sample of diverse depressed patients.

\subsection{Panic disorder and agoraphobia}

Panic disorder and/or agoraphobia were most highly prevalent in the Novelty seekers cluster, and their lowest occurrence was observed in the Persistent cluster. Considering the specifications of panic disorder 
and agoraphobia in the Diagnostic and Statistical Manual of mental disorders (DSM-IV-TR (2000) 4th ed., text rev.), these disorders likely associate with both types of anxiety defined in Cloninger's original theory, namely cognitive and somatic, with panic attacks responding to somatic anxiety and continuous concern of attacks or agoraphobia to cognitive anxiety. Thus, the anticipated result was that the highest prevalence of PDA would be observed in the Novelty seekers cluster, which was characterized by highest scores in NS (presumably associated with somatic anxiety) and above the population norm in HA (presumably associated with cognitive anxiety) (Jylhä and Isometsä, 2006). In the logistic regression models (adjusted with depression severity, alcohol use, age and gender), the odds for PDA for Novelty seekers were over three times as high as that for other patients, thereby suggesting a strong association between the Novelty seekers temperament profile and panic disorder. Earlier evidence has mainly suggested no associations between the trait 'high NS' and panic disorder comorbidity in depressed patients and one earlier study has found an inverse relation between the two (Ampollini et al., 1999; Kennedy et al., 2001; Ongur et al., 2005). However, these studies likely included patient samples with less variance in NS compared with our sample due to excluding patients with substance use disorders (Ampollini et al., 1999; Kennedy et al., 2001; Ongur et al., 2005), who 1) are likely to have higher NS scores (Howard et al., 1997) and 2) have a high predisposition to panic disorder (Zvolensky et al., 2006). This difference in samples could explain our novel finding of a strong association between Novelty seekers temperament (with high NS) and higher risk for panic disorder.

Neuropsychologically the increased morbidity in panic disorder and agoraphobia in Novelty seekers could be associated with aberrant functioning in paralimbic brain areas that are associated with both Novelty Seeking and panic disorder (De Cristofaro, et al, 1993; Sugiura, et al, 2000). These brain areas including right anterior cingulate cortex and anterior/posterior insula constitute in part networks associated with interoceptive functions and with panic disorder (Cui, et al, 2016; LeDoux and Pine, 2016; Sugiura, et al, 2000). Psychological theories have also suggested that interoceptive functions (e.g. interoceptive conditioning and/or catastrophic misappraisals of bodily sensations) are central in the etiology of panic attacks and panic disorder (Bouton, et al, 2001; Craske and Waters, 2005). High Novelty Seeking could be a 
moderator of the association between interoceptive functions and panic disorder hypothetically via "histrionic" information processing style (Cloninger, 1986). This kind of moderating effect would be in line with our finding of highest prevalence of PDA in patients with a temperament profile including the highest NS scores (Novelty seekers) and significantly lower prevalence in patients with a temperament profile including the lowest NS scores (Reserved).

Earlier studies have shown that panic disorder is associated with high HA (Kampman et al., 2014), and in addition to high NS observed in Novelty seekers, increased HA could be an important part of this temperament profile, thus explaining the high prevalence of panic disorder (and SAD) found in this patient group.

\subsection{Social anxiety disorder}

The highest prevalence of social anxiety disorder was observed in the Wearied cluster and the lowest in the Persistent cluster. After adjusting with clinical variables, the Wearied temperament profile, characterized by highest $H A$ and lowest RD and $P$, predicted $S A D$ in the regression models with a higher odds that was over three times as high as that for other patients. Earlier evidence has shown that high HA is associated with all anxiety disorders with highest levels found in SAD, which is well in line with our finding of Wearied cluster patients (having the highest HA) having the high prevalence of different anxiety disorders and the highest prevalence of SAD (Kampman et al., 2014; Miettunen and Raevuori, 2012). HA is also associated with severity of depressive symptoms and all these findings together suggest that the highest scores in HA are associated with SAD and more severe depression (Kampman and Poutanen, 2011). According to high HA's definition, this trait is associated with avoidant behavior and according to Cloninger's original theory, high HA is also associated with obsessional information processing type (Cloninger, 1986; Cloninger, 1987). These kinds of behavioral avoidance and cognitive unconstructive repetitive thinking patterns could be the link between high HA and both SAD and depression (Kanter et al., 2008; Watkins, 2008; Weinstock and Whisman, 2007). This would lead to interpreting the preceding findings as suggesting that more severe depression and SAD are more strongly associated with avoidance and ruminative or repetitive thinking patterns compared with other anxiety disorders. This kind of common etiological factor could explain the 
higher comorbidity rate of SAD compared with other anxiety disorders in MDD patients (Brown et al., 2001) and suggest that the etiology of SAD resembles more to that of severe depression than other anxiety disorders in these cognitive and behavioral aspects. As negative mood valent rumination is associated with heightened neural activity in default mode network (Graham et al., 2013; Malhi et al., 2015), high HA could be linked to depression via similar neuropsychological mechanisms.

Negative affectivity or neuroticism, which represent traits similar to HA (Capanna et al., 2012), have been proposed by earlier studies as posing a higher-order personality trait common to depressive and anxiety disorders and that other unique factors differentiate between separate disorders (Clark and Watson, 1991; Craske and Waters, 2005). Although the highest scores of HA could be associated especially with SAD and more severe depression, as discussed in a previous paragraph, HA is likely to also present more general factors predisposing to psychopathology, similarly to neuroticism (Kampman and Poutanen, 2011; Kampman et al., 2014; Miettunen and Raevuori, 2012). Moreover, our results suggest that emotional detachment and low ambition (reflected as lower RD and P in the Wearied patients' temperament profile) compared with other patients could be an important distinctive characteristic of this temperament profile associated specifically with higher risk for SAD.

\subsection{Generalized anxiety disorder}

Generalized anxiety disorder had the highest prevalence in the Reserved cluster and the lowest in the Persistent cluster. GAD was predicted by the Reserved temperament profile in the regression models with over two times as high odds than that of other patients. Reserved patients were characterized by lowest $\mathrm{NS}$, high $\mathrm{HA}$, and had RD and $\mathrm{P}$ traits roughly corresponding to those observed in the Finnish general population (Jylhä and Isometsä, 2006). Evidence of the associations between temperament and GAD is scarce, but the main finding has been in line with our results, with GAD suggested to be associated with high HA (Kampman et al., 2014). In addition to this, our results suggest that low NS could be an important part of the temperament profile associated with GAD even though a similar association has not been found previously (Ongur et al., 2005; Piero, 2010). As discussed in chapter 4.1, panic disorder is suggested to be associated with interoceptive functions and Novelty seekers temperament profile (with high NS responding 
to "histrionic" information processing) could be a moderator of this association. Thus, Reserved patients could be at lower risk for panic disorder due to being less affected with "histrionic" or impulsive information processing. Instead, the present results suggest that the proclivity to slow and rigid decision making, slow engagement to new interests or preoccupation in details associated with low NS could predispose these individuals to generalized anxiety when experienced together with other traits predisposing to development of psychopathology, such as high HA (Cloninger, 1987; Miettunen and Raevuori, 2012).

\subsection{Persistent temperament profile}

The lowest prevalence in all analyzed anxiety disorders was associated with the Persistent cluster which was characterized by highest P and lowest HA when compared to the other temperament clusters. Moreover, the Persistent patients could likely be associated with higher $\mathrm{P}$ when compared to general population because markedly lower scores $(P=114.6 \pm 17.3)$ have been found in Finnish general population earlier (Jylhä and Isometsä, 2006). However, the lack of a control group prevents conclusions of the relative differences between these populations. Finding the Persistent patients having lower prevalence of anxiety disorders was a plausible result because high $\mathrm{P}$ has been associated with positive emotionality and wellbeing (Cloninger et al., 1998; Garcia, 2011), especially together with low HA and character trait low SelfDirectedness (Cloninger et al., 2012). Our results are in line with these findings and support a hypothesis that the combination of low HA and high P associates with lower comorbidity of anxiety disorders in depressed patients.

\subsection{Strengths and limitations}

Because individual temperament presents a precognitive bias resulting in different behaviors, the cluster analysis provided complete temperament profiles reflecting more comprehensively the individual, temperament-oriented behavior in patients. Although meta-analytic data exist on how different temperament traits are uniquely predisposing to different affective disorders, it was important to test the hypothesis of whether individual complete profile data (in terms of temperament clusters) predict the 
occurrence of anxiety disorders in depressed patients. Moreover, it is possible that these temperament clusters would reflect endophenotypes predisposing to different disorders because they were associated with different disorders in this study. These kinds of endophenotypes could be more closely associated with the biological background of psychiatric symptoms compared with categorical diagnoses or separate temperament traits and could help in the progress of finding biomarkers for psychopathology (Service et al., 2012). Testing the possible associations between temperament clusters and candidate genes of anxiety disorders would be interesting in future studies (Sharma et al., 2016).

Although the temperament profiles of the studied four temperament clusters showed strong associations with different anxiety disorders, the lack of a control group in the study posits some limitations that have to be considered when interpreting the results. Firstly, the four temperament clusters may or may not be a unique feature of depressed populations and studies with non-depressed controls are needed to give more insight on this question. Secondly, the patients' temperament profile was assessed during depressive symptoms and may not represent their long-term temperament profile because depressive states are known to be associated with elevation in temperament trait Harm Avoidance (Kampman and Poutanen, 2011). However, the main focus of this study was to evaluate the associations between temperament clusters and the occurrence of anxiety comorbidities. In these analyses the depression severity was controlled for to avoid the bias towards higher risk of comorbidities with more severe depression. Thirdly, because of the high level of comorbid disorders in our sample it is also possible that the current finding of the four temperament clusters is specific for depression with anxiety disorder and substance use comorbidities. Moreover, although human temperament is considered relatively stable over one's life-span (Cloninger et al., 1993) and the temperament clusters predicted specifically higher risk for different anxiety disorders in this study, the cross-sectional setting does not allow interpretations of the possible causal relations between the temperament profiles and the studied anxiety disorders. Longitudinal studies in general population are needed for making conclusions of the possible causality and could have more marked clinical implications compared to studies with cross-sectional settings. Due to the study design using cluster analysis for creating temperament clusters and aiming at analyzing their associations with 
anxiety disorders, we had no a priori hypotheses in this study, which may also be considered to be a limitation. The use of a semi-structured MINI interview (based on the DSM-IV) could have resulted in overdiagnosing the patients with SAD and PDA because no exclusion criteria according to the hierarchy of these diagnoses were used. However, the diagnosis of GAD was excluded in cases of any other anxiety comorbidities. We combined patients with panic disorder and/or agoraphobia into one group (PDA), which limits the generalizability of these results to clinical settings. Moreover, when interpreting the results, it must also be noted that 25 patients had undergone a previous hypomanic episode, which limits the generalizability of these results to MDD patients. Instead, the results reflect more of the associations between temperament profiles and more diverse patients with current depressive symptoms.

\subsection{Conclusions}

Temperament clusters with unique dimensional profiles were specifically associated with different anxiety disorders in this study. These results suggest that TCI-R could offer a valuable dimensional method for predicting the risk of anxiety disorders in diverse depressed patients.

\section{Acknowledgments}

This study was supported by a grant from Finland's Psychiatric Research Association and Finnish Psychiatric Association (Dr. Paavonen) and funds from Southern Ostrobothnia Hospital District (EVO1114), University of Tampere and State of Finland. Funding sources were not involved in the study design process or writing of the article. The authors express their warmest thanks to research nurses Susanna Ahola, Marja Koivumäki, and Kati Huhtala for their skillful efforts and to all participants for their collaboration during the study. We thank Edanz Group (www.edanzediting.com/ac) for editing a draft of this manuscript. 


\section{References}

Al-Halabi, S., Herrero, R., Saiz, P.A., Garcia-Portilla, M.P., Corcoran, P., Bascaran, M.T., Errasti, J.M., Lemos, S., Bobes, J., 2010. Sociodemographic factors associated with personality traits assessed through the TCl. Personality and Individual Differences. 48, 809-814.

Ampollini, P., Marchesi, C., Signifredi, R., Ghinaglia, E., Scardovi, F., Codeluppi, S., Maggini, C., 1999. Temperament and personality features in patients with major depression, panic disorder and mixed conditions. J. Affect. Disord. 52, 203-207.

Barlow, D.H. and Kennedy, K.A., 2016. New Approaches to Diagnosis and Treatment in Anxiety and Related Emotional Disorders: A Focus on Temperament. Canadian Psychology. 57, 8-20.

Beck, A.T., Steer, R.A., Ball, R., Ranieri, W., 1996. Comparison of Beck Depression Inventories -IA and -II in psychiatric outpatients. J. Pers. Assess. 67, 588-597.

Bereczkei, T. and Czibor, A., 2014. Personality and situational factors differently influence high Mach and low Mach persons' decisions in a social dilemma game. Personality and Individual Differences. 64, 168-173.

Bohn, M.J., Babor, T.F., Kranzler, H.R., 1995. The Alcohol Use Disorders Identification Test (AUDIT): validation of a screening instrument for use in medical settings. J. Stud. Alcohol. 56, 423-432.

Bouton, M.E., Mineka, S., Barlow, D.H., 2001. A Modern Learning Theory Perspective on the Etiology of Panic Disorder. Psychol. Rev. 108, 4-32.

Brown, T.A., Campbell, L.A., Lehman, C.L., Grisham, J.R., Mancill, R.B., 2001. Current and Lifetime Comorbidity of the DSM-IV Anxiety and Mood Disorders in a Large Clinical Sample. J. Abnorm. Psychol. 110, 585-599.

Burcusa, S.L. and lacono, W.G., 2007. Risk for recurrence in depression. Clin. Psychol. Rev. 27, 959-985.

Campbell, N., Eley, D., McAllister, L., 2013. What does personality tell us about working in the bush? Temperament and character traits of Australian remote allied health professionals. Aust. J. Rural Health. $21,240-248$.

Capanna, C., Struglia, F., Riccardi, I., Daneluzzo, E., Stratta, P., Rossi, A., 2012. Temperament and Character Inventory-R (TCI-R) and Big Five Questionnaire (BFQ): convergence and divergence. Psychol. Rep. 110, 1002-1006.

Cheavens, J.S. and Heiy, J., 2011. The differential roles of affect and avoidance in major depressive and borderline personality disorder symptoms. Journal of Social and Clinical Psychology. 30, 441-457.

Clark, L.A. and Watson, D., 1991. Tripartite Model of Anxiety and Depression: Psychometric Evidence and Taxonomic Implications. Journal of Abnormal Psychology.Special Issue on Diagnoses, Dimensions, and DSMIV: The Science of Classification. 100, 316-336.

Cloninger, C.R., Bayon, C., Svrakic, D.M., 1998. Measurement of temperament and character in mood disorders: a model of fundamental states as personality types. J. Affect. Disord. 51, 21-32. 
Cloninger, C.R., Zohar, A.H., Hirschmann, S., Dahan, D., 2012. The psychological costs and benefits of being highly persistent: personality profiles distinguish mood disorders from anxiety disorders. J. Affect. Disord. $136,758-766$.

Cloninger, C.R., 1986. A unified biosocial theory of personality and its role in the development of anxiety states. Psychiatr. Dev. 4, 167-226.

Cloninger, C.R., 1987. A systematic method for clinical description and classification of personality variants: A proposal. Arch. Gen. Psychiatry. 44, 573-588.

Cloninger, C.R., Svrakic, D.M., Przybeck, T.R., 1993. A Psychobiological Model of Temperament and Character. Arch. Gen. Psychiatry. 50, 975-990.

Craske, M.G. and Waters, A.M., 2005. Panic disorder, phobias, and generalized anxiety disorder. Annu. rev. clin. psychol. 1, 197-225.

Cui, H., Zhang, J., Liu, Y., Li, Q., Li, H., Zhang, L., Hu, Q., Cheng, W., Luo, Q., Li, J., Li, W., Wang, J., Feng, J., Li, C., Northoff, G., 2016. Differential alterations of resting-state functional connectivity in generalized anxiety disorder and panic disorder. Hum. Brain Mapp. 37, 1459-1473.

De Cristofaro, M.T., Sessarego, A., Pupi, A., Biondi, F., Faravelli, C., 1993. Brain perfusion abnormalities in drug-naive, lactate-sensitive panic patients: a SPECT study. Biol. Psychiatry. 33, 505-512.

El Sheikh, M.M., Shaker, N.M., Hussein, H., Ramy, H.A., 2014. Impact of personality temperaments and characters on academic performance and specialty selection among a group of Egyptian medical graduates. Int. J. Soc. Psychiatry. 60, 499-507.

Fernandez-Mondragon, S. and Adan, A., 2015. Personality in male patients with substance use disorder and/or severe mental illness. Psychiatry Res. 228, 488-494.

Garcia, D., 2011. Two models of personality and well-being among adolescents. Personality and Individual Differences. 50, 1208-1212.

Graham, J., Salimi-Khorshidi, G., Hagan, C., Walsh, N., Goodyer, I., Lennox, B., Suckling, J., 2013. Metaanalytic evidence for neuroimaging models of depression: state or trait? J. Affect. Disord. 151, 423-431.

Holzel, L., Harter, M., Reese, C., Kriston, L., 2011. Risk factors for chronic depression--a systematic review. J. Affect. Disord. 129, 1-13.

Howard, M.O., Kivlahan, D., Walker, R.D., 1997. Cloninger's tridimensional theory of personality and psychopathology: applications to substance use disorders. J. Stud. Alcohol. 58, 48-66.

Jylhä, P. and Isometsä, E., 2006. Temperament, character and symptoms of anxiety and depression in the general population. European Psychiatry. 21, 389-395.

Kampman, O. and Poutanen, O., 2011. Can onset and recovery in depression be predicted by temperament? A systematic review and meta-analysis. J. Affect. Disord. 135, 20-27.

Kampman, O., Viikki, M., Jarventausta, K., Leinonen, E., 2014. Meta-analysis of anxiety disorders and temperament. Neuropsychobiology. 69, 175-186. 
Kanter, J.W., Busch, A.M., Weeks, C.E., Landes, S.J., 2008. The nature of clinical depression: symptoms, syndromes, and behavior analysis. Behav. Anal. 31, 1-21.

Kennedy, B.L., Schwab, J.J., Hyde, J.A., 2001. Defense styles and Personality dimensions of research subjects with anxiety and depressive disorders. Psychiatr. Q. 72, 251-262.

Klein, D.N., Kotov, R., Bufferd, S.J., 2011. Personality and depression: explanatory models and review of the evidence. Annu. rev. clin. psychol. 7, 269-295.

LeDoux, J.E. and Pine, D.S., 2016. Using neuroscience to help understand fear and anxiety: A two-system framework. Am. J. Psychiatry. 173, 1083-1093.

Malhi, G.S., Byrow, Y., Fritz, K., Das, P., Baune, B.T., Porter, R.J., Outhred, T., 2015. Mood disorders: neurocognitive models. Bipolar Disord. 17, 3-20.

Melartin, T.K., Rytsala, H.J., Leskela, U.S., Lestela-Mielonen, P.S., Sokero, T.P., Isometsa, E.T., 2002. Current comorbidity of psychiatric disorders among DSM-IV major depressive disorder patients in psychiatric care in the Vantaa Depression Study. J. Clin. Psychiatry. 63, 126-134.

Mezulis, A.H., Priess, H.A., Hyde, J.S., 2011. Rumination Mediates the Relationship between Infant Temperament and Adolescent Depressive Symptoms. Depress Res. Treat. 2011, 487873.

Miettunen, J. and Raevuori, A., 2012. A meta-analysis of temperament in axis I psychiatric disorders. Comprehensive Psychiatry. 53, 152-166.

Montgomery, S.A. and Asberg, M., 1979. A new depression scale designed to be sensitive to change. Br. J. Psychiatry. 134, 382-389.

Naragon-Gainey, K., Prenoveau, J.M., Brown, T.A., Zinbarg, R.E., 2016. A Comparison and Integration of Structural Models of Depression and Anxiety in a Clinical Sample: Support for and Validation of the Tri-Level Model. J. Abnorm. Psychol. 125, 853-867.

Ongur, D., Farabaugh, A., losifescu, D.V., Perlis, R., Fava, M., 2005. Tridimensional Personality Questionnaire Factors in Major Depressive Disorder: Relationship to Anxiety Disorder Comorbidity and Age of Onset. Psychotherapy \& Psychosomatics. 74, 173-178.

Otani, K., Suzuki, A., Ishii, G., Matsumoto, Y., Kamata, M., 2008. Relationship of interpersonal sensitivity with dimensions of the Temperament and Character Inventory in healthy subjects. Compr. Psychiatry. 49, 184-187.

Paavonen, V., Luoto, K., Koivukangas, A., Lassila, A., Leinonen, E., Kampman, O., 2016. Temperament and character profiles associated with depression and treatment response in patients with or without comorbid substance abuse. Psychiatry Res. 245, 250-258.

Piero, A., 2010. Personality correlates of impulsivity in subjects with generalized anxiety disorders. Compr. Psychiatry. 51, 538-545.

Rae, A.M., Joyce, P.R., Luty, S.E., Mulder, R.T., 2002. The effect of a history of alcohol dependence in adult major depression. Journal of Affective Disorders. 70, 281-290. 
Service, S.K., Verweij, K.J.H., Lahti, J., Congdon, E., Ekelund, J., Hintsanen, M., Raikkonen, K., Lehtimaki, T., Kahonen, M., Widen, E., Taanila, A., Veijola, J., Heath, A.C., Madden, P.A.F., Montgomery, G.W., Sabatti, C., Jarvelin, M., Palotie, A., Raitakari, O., Viikari, J., Martin, N.G., Eriksson, J.G., Keltikangas-Jarvinen, L., Wray, N.R., Freimer, N.B., 2012. A genome-wide meta-analysis of association studies of Cloninger's Temperament Scales. Transl. Psychiatry. 2, e116.

Sharma, S., Powers, A., Bradley, B., Ressler, K.J., 2016. Gene x Environment Determinants of Stress- and Anxiety-Related Disorders. Annu. Rev. Psychol. 67, 239-261.

Sheehan, D.V., Lecrubier, Y., Sheehan, K.H., Amorim, P., Janavs, J., Weiller, E., Hergueta, T., Baker, R., Dunbar, G.C., 1998. The Mini-International Neuropsychiatric Interview (M.I.N.I.): the development and validation of a structured diagnostic psychiatric interview for DSM-IV and ICD-10. J. Clin. Psychiatry. 59, 2233;quz.

Sher, K.J., Bartholow, B.D., Wood, M.D., 2000. Personality and Substance Use Disorders: A Prospective Study. Journal of Consulting \& Clinical Psychology. 68, 818-829.

Starcevic, V., Uhlenhuth, E.H., Fallon, S., Pathak, D., 1996. Personality dimensions in panic disorder and generalized anxiety disorder. J. Affect. Disord. 37, 75-79.

Sugiura, M., Kawashima, R., Nakagawa, M., Okada, K., Sato, T., Goto, R., Sato, K., Ono, S., Schormann, T., Zilles, K., Fukuda, H., 2000. Correlation between human personality and neural activity in cerebral cortex. Neuroimage. 11, 541-546.

Watkins, E.R., 2008. Constructive and unconstructive repetitive thought. Psychol. Bull. 134, 163-206.

Weinstock, L.M. and Whisman, M.A., 2007. Rumination and excessive reassurance-seeking in depression: A cognitive-interpersonal integration. Cognitive Therapy and Research. 31, 333-342.

Zvolensky, M.J., Bernstein, A., Marshall, E.C., Feldner, M.T., 2006. Panic attacks, panic disorder, and agoraphobia: Associations with substance use, abuse, and dependence. Curr. Psychiatry Rep. 8, 279-285.

American Psychiatric Association. (2000). Diagnostic and Statistical

Manual of Mental Disorders (4th ed., text rev.).

https://dsm.psychiatryonline.org/doi/pdf/10.1176/appi.books.9780890420249.dsm-iv-tr, (accessed:

December 21, 2017)

Ostrobothnia Depression Study (ODS). A Naturalistic Follow-up Study on Depression and Related Substance Use Disorders. https://clinicaltrials.gov/ct2/show/NCT02520271, (accessed: December 21, 2017) 
Table 1. Clinical characteristics and distributions of anxiety disorders and their differences between the four temperament clusters (Novelty seekers,

Persistent, Reserved and Wearied).

\begin{tabular}{|c|c|c|c|c|c|c|c|c|c|}
\hline \multirow[b]{2}{*}{$\begin{array}{l}\text { Temperament } \\
\text { clusters }\end{array}$} & \multicolumn{6}{|c|}{ Clinical variables } & \multicolumn{3}{|c|}{ Diagnoses } \\
\hline & $\begin{array}{c}\text { Age * } \\
(\text { mean } \pm S D)\end{array}$ & $\begin{array}{l}\text { Male } \\
\text { gender } \\
(n, \%)\end{array}$ & $\begin{array}{l}\text { Antipsychotics } \\
\text { in use }\end{array}$ & $\begin{array}{c}\text { Antidepressant } \\
\text { dose (mean } \pm S D \text { ) }\end{array}$ & $\begin{array}{c}\text { AUDIT-C } \\
\text { scores } \\
\text { (mean } \pm S D)\end{array}$ & $\begin{array}{c}\text { MADRS } \\
\text { scores } \\
\text { (mean } \pm S D)\end{array}$ & $\begin{array}{l}\text { PDA } \\
(n, \%)\end{array}$ & $\begin{array}{l}\text { SAD } \\
(n, \%)\end{array}$ & $\begin{array}{l}\text { GAD } \\
(n, \%)\end{array}$ \\
\hline $\begin{array}{l}\text { 2. Persistent } \\
\left(n=36,17 \%^{* *}\right)\end{array}$ & $42.5 \pm 12.2$ & $12,33.3 \%$ & $7,19.4 \%$ & $25.2 \pm 18.7$ & $4.31 \pm 3.43$ & $23.1 \pm 7.7$ & $5,14.3 \%$ & $1,2.9 \%$ & $8,22.9 \%$ \\
\hline $\begin{array}{l}\text { 3. Reserved } \\
(n=66,30 \% * *)\end{array}$ & $41.4 \pm 11.6$ & $22,33.3 \%$ & $15,22.7 \%$ & $25.0 \pm 21.0$ & $4.39 \pm 3.29$ & $22.6 \pm 6.1$ & $14,22.2 \%$ & $10,15.9 \%$ & $20,31.7 \%$ \\
\hline $\begin{array}{l}\text { 4. Wearied } \\
\left(n=58,27 \%^{* *}\right)\end{array}$ & $34.8 \pm 11.4$ & $25,43.1 \%$ & $22,37.9 \%$ & $32.4 \pm 19.2$ & $4.93 \pm 3.24$ & $24.5 \pm 5.4$ & $22,40.0 \%$ & $19,34.5 \%$ & $6,10.9 \%$ \\
\hline \multicolumn{10}{|l|}{$\begin{array}{l}\text { Difference between } \\
\text { the clusters }\end{array}$} \\
\hline
\end{tabular}

Abbreviations: AUDIT-C = Alcohol use disorder identification test (questions 1-3); PDA = Panic disorder and/or Agoraphobia; SAD = Social anxiety disorder;

$\mathrm{GAD}=$ Generalized anxiety disorder

Percentages in clinical variables and diagnoses are proportions of the temperament clusters

*differences were significant between clusters 2 and 4, and between clusters 3 and 4 in Bonferroni analysis

**proportion of the whole patient sample

${ }^{1}$ for ANOVA between the temperament clusters

${ }^{2}$ for chi-square between the temperament clusters

Significant differences are in boldface 
Table 2. Likelihood of anxiety disorders (PDA, SAD and GAD) according to the temperament clusters in the logistic regression models.

\begin{tabular}{|c|c|c|c|c|c|c|c|c|c|c|c|c|}
\hline & \multicolumn{4}{|c|}{ PDA } & \multicolumn{4}{|c|}{ SAD } & \multicolumn{4}{|c|}{ GAD } \\
\hline & \multirow[t]{2}{*}{ OR } & \multicolumn{2}{|c|}{$95 \% \mathrm{Cl}$} & \multirow[t]{2}{*}{$p$} & \multirow[t]{2}{*}{ OR } & \multicolumn{2}{|c|}{$95 \% \mathrm{Cl}$} & \multirow[t]{2}{*}{$p$} & \multirow[t]{2}{*}{ OR } & \multicolumn{2}{|c|}{$95 \% \mathrm{Cl}$} & \multirow[t]{2}{*}{$p$} \\
\hline & & lower & upper & & & lower & upper & & & lower & upper & \\
\hline \multicolumn{13}{|l|}{ Model 1} \\
\hline $\begin{array}{l}\text { Temperament } \\
\text { cluster* }\end{array}$ & 3.28 & 1.67 & 6.41 & 0.001 & 3.17 & 1.49 & 6.76 & 0.003 & 2.60 & 1.27 & 5.33 & 0.009 \\
\hline \multicolumn{13}{|l|}{ Model 2} \\
\hline $\begin{array}{l}\text { Temperament } \\
\text { cluster* }\end{array}$ & 3.52 & 1.78 & 6.96 & $<0.001$ & 3.19 & 1.47 & 6.90 & 0.003 & 2.53 & 1.23 & 5.21 & 0.01 \\
\hline \multicolumn{13}{|l|}{ Model 3} \\
\hline $\begin{array}{l}\text { Temperament } \\
\text { cluster* }\end{array}$ & 3.48 & 1.76 & 6.91 & $<0.001$ & 3.41 & 1.55 & 7.49 & 0.002 & 2.55 & 1.24 & 5.27 & 0.01 \\
\hline
\end{tabular}

Model 1: adjusted with gender and age

Model 2: adjusted with gender and age, and MADRS scores

Model 3: adjusted with gender, age, MADRS scores and AUDIT-C scores

*Binominal variables: 1) Novelty seekers cluster vs. others explaining PDA, 2) Wearied cluster vs. others explaining $S A D$, and 3) Reserved cluster vs. others explaining GAD

Abbreviations: PDA = Panic disorder and/or agora phobia; SAD = Social anxiety disorder; GAD = General anxiety disorder; MADRS = Montgomery Åsberg depression rating scale; AUDIT-C $=$ Alcohol use disorder identification test (questions $1-3$ ), $\mathrm{OR}=$ odds ratio, $\mathrm{Cl}=$ confidence interval 
Supplementary Table. MADRS and AUDIT-C scores, and sociodemographic data of the patient sample

\begin{tabular}{|c|c|c|}
\hline & $N$ & $\%$ \\
\hline Total & 204 & 100 \\
\hline \multicolumn{3}{|l|}{ Marital status } \\
\hline Single & 65 & 32 \\
\hline Married or cohabiting & 99 & 49 \\
\hline Divorced & 38 & 19 \\
\hline Widowed & 2 & 1 \\
\hline \multicolumn{3}{|l|}{ Education } \\
\hline Primary school & 6 & 3 \\
\hline Comprehensive school & 48 & 24 \\
\hline Tertiary education & 30 & 15 \\
\hline Vocational school & 77 & 38 \\
\hline Upper secondary education & 17 & 8 \\
\hline Polytechnic or university & 26 & 13 \\
\hline \multicolumn{3}{|l|}{ Work status before sick leave } \\
\hline Employed & 99 & 49 \\
\hline Unemployed & 62 & 31 \\
\hline Housewife/husband & 8 & 4 \\
\hline Pensioner & 14 & 7 \\
\hline Student & 19 & 9 \\
\hline $\begin{array}{l}\text { Self-reported history of } \\
\text { depressive episode }\end{array}$ & 134 & 66 \\
\hline $\begin{array}{l}\text { First degree family history of } \\
\text { depression }\end{array}$ & 79 & 39 \\
\hline \multirow[t]{2}{*}{$\begin{array}{l}\text { First degree family history of } \\
\text { bipolar disorder }\end{array}$} & 13 & 6 \\
\hline & Mean & $\pm \mathrm{SD}$ \\
\hline MADRS scores & 23.2 & 6.3 \\
\hline AUDIT-C scores & 4.8 & 3.4 \\
\hline
\end{tabular}

Abbreviations: MADRS = Montgomery Åsberg depression rating scale; AUDIT-C = Alcohol use disorder identification test (questions 1-3) 


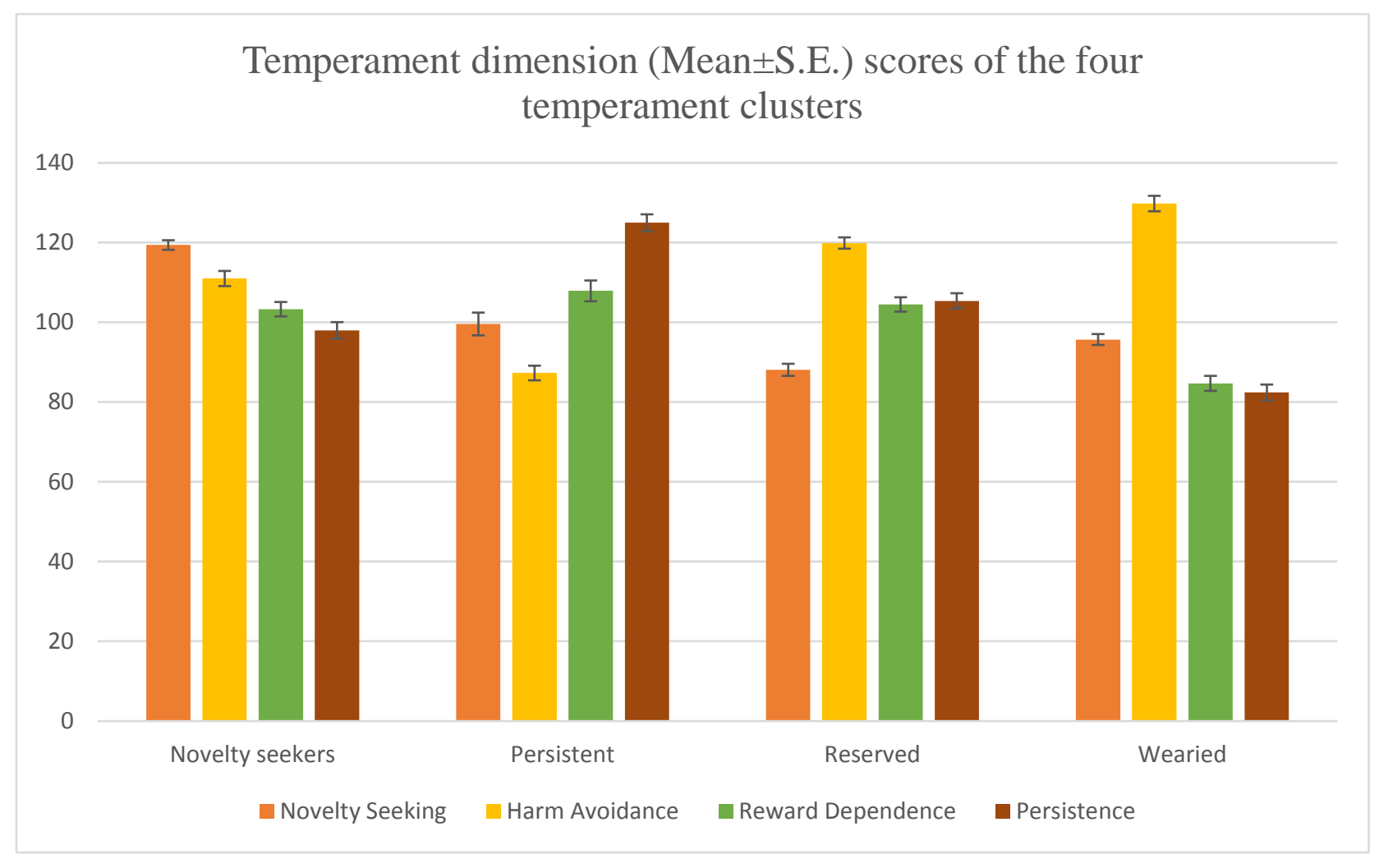

